\title{
WILEY-VCH
}

DOI: 10.1002/adma.201504015

Article type: Communication

\section{Magnetic Nanocomposite Cilia Tactile Sensor}

Ahmed Alfadhel, and Jürgen Kosel*

\author{
A. Alfadhel, J. Kosel \\ Computer, Electrical and Mathematical Sciences and Engineering Division (CEMSE), King \\ Abdullah University of Science and Technology (KAUST), Saudi Arabia \\ E-mail: jurgen.kosel@kaust.edu.sa
}

Keywords: tactile sensors, nanocomposites, nanowires, magnetic sensors, cilia

The evolution in areas like robotics is demanding an increased perception of the environment such as touch, vibration and flow sensing. Noticeable progress in the field of artificial skins lead to the development of different technologies that can mimic the complex sense of touch in humans for better interaction with the surrounding environment. These skins are also important for applications like wearable consumer electronics or health monitoring systems, ${ }^{[1-7]}$ smart surgical tools, ${ }^{[8]}$ or to provide the sensitivity missing in prosthetics to enable people with artificial arms or legs to "feel" the world around them again. ${ }^{[9]}$ Tactile sensors are the essential components for artificial skins, and different physical principles have been exploited for the development of highly sensitive and low power tactile sensors realized on flexible substrates allowing conformal coverage of electronic systems on nonplanar surfaces, and hence enabling new functionalities. ${ }^{[10,11]}$ A tactile sensing mechanism introduced by Gong et.al, ${ }^{[12]}$ is based on the resistivity of a gold nanowires nanocomposite that has shown a high resolution of $13 \mathrm{~Pa}$ with a sensing range up to $50 \mathrm{kPa}$. It has the ability to detect bending, torsion and pressing with a power consumption of $30 \mu \mathrm{W}$. Other resistive tactile sensors developed by Zhu et. al were made from a micro-structured graphene/PDMS nanocomposite showing an ultra-high sensitivity of $1.5 \mathrm{~Pa}$ with a sensing range up to $1.5 \mathrm{kPa} \cdot{ }^{[13]}$ The sensors can detect vertical pressure and provide a very fast dynamic response of $0.2 \mathrm{~ms}$. A piezoresistive tactile sensor 


\section{WILEY-VCH}

was proposed by Yilmazoglu et. al. ${ }^{[14]}$ It uses carbon nanotubes, has a resolution of $32 \mathrm{mN}$ and operates with power consumption of $1.9 \mu \mathrm{W}$. Different capacitive tactile sensors have been presented in literature such as the capacitive sensor with microstructures rubber dielectric proposed by Mannsfeld, et. al that can detect a low pressure of $3 \mathrm{~Pa}$ and operates up to $7 \mathrm{kPa} .^{[15]}$ A highly skin-conformal cilia capacitive sensor has shown outstanding performance for pulse signal amplification and a high potential for wearable health monitoring systems. ${ }^{[16,17]}$ Many other tactile sensing concepts have been introduced in literature that have helped the progression of the tactile sensing technology. ${ }^{[18-23]}$ Despite this rapid development, there are still many challenges that need to be addressed. It is especially challenging to combine a high resolution with a low power consumption while maintaining multi-functionality (sensing of flow, vibration, touch, ....) and the ability to operate in different media such as water and air. Artificial cilia sensors are devices developed to mimic the extremely sensitive mechanosensorial hair-like cilia receptors found in nature. ${ }^{[16,24]}$ For instance, cilia are used as the touch and vibration sensing receptors on insect legs, ${ }^{[25]}$ the cerci of crickets for flow sensing, ${ }^{[26]}$ and the cochlea in the inner ear. ${ }^{[27]}$ They enable transferring of various mechanical forces and provide exquisite sensing performance, mainly due to the high aspect ratio and high surface area to volume ratio, which ensures strong interaction with the environment.

We report the development of highly elastic and permanent magnetic nanocomposite artificial cilia integrated on a magnetic sensing element as a novel tactile sensing approach (Figure 1(a)). The nanocomposite is made of iron nanowires (NWs) incorporated into polydimethylsiloxane (PDMS). The cilia utilize the permanent magnetic behavior of the NWs, allowing remote operation without an additional magnetic field to magnetize the NWs, which minimizes the power consumption and simplifies system integration. In addition, the nanocomposite offers a high elasticity, easy patterning and corrosion resistance. Employing this concept, we realize highly sensitive, power efficient and multifunctional tactile sensors which can operate in air 


\section{WILEY-VCH}

and liquid. The resolution, sensitivity and operating range can be easily tuned with the dimensions of the cilia to accommodate a wide range of applications.

The operating principle of the sensor is based on detecting the change of the cilia's magnetic field, created by the iron NWs when deflected by an external force (e.g. vibration, fluid flow or hand touch). A multi-layer giant magneto-impedance (GMI) sensor, which offers a high sensitivity and a simple fabrication process, ${ }^{[28-31]}$ is utilized to measure the change of the magnetic field. A distinct advantage of the nanocomposite cilia is the permanent magnetic behavior of the iron NWs, which provides the required bias field for the magnetic sensor. In the presence of a force, the cilia bend, resulting in a change of the average magnetic field value affecting the GMI sensor, and hence changing its impedance.

The proposed concept has wide range of flexibility to achieve: a sensor with extremely high sensitivity within an ultra-low-pressure regime $(<500 \mathrm{~Pa})$ for microfluidics or biological detection, a sensor with high degree of sensitivity in the low-pressure regime $(<10 \mathrm{kPa})$ for electronic skins and health monitoring systems, or a sensor with wide range of operation up to $300 \mathrm{kPa}$ for industrial applications.

The NWs-PDMS nanocomposite serves as the structural material for the cilia and is prepared by incorporating $6 \mu \mathrm{m}$ long and $35 \mathrm{~nm}$ in diameter iron NWs into PDMS. Iron NWs are chosen for their high magnetization at remanence and high coercivity, making them nano-permanent magnets, in addition to their biocompatibility. ${ }^{[32]}$ It has previously been shown that the nanocomposite has a good corrosion resistance, protecting the NWs from fast oxidation. ${ }^{[33]}$ The cilia are integrated on GMI sensors fabricated on silicon or Kapton substrates for rigid or flexible tactile sensors, respectively.

The GMI sensors are made of a $200 \mathrm{~nm}$ thick $\mathrm{Cu}$ layer sandwiched by two $100 \mathrm{~nm}$ thick $\mathrm{Ni}_{80} \mathrm{Fe}_{20}$ layers. They have a meander structure of $4 \mathrm{~mm}^{2}$ composed of $2 \mathrm{~mm}$ long and $200 \mu \mathrm{m}$ wide conductors with $200 \mu \mathrm{m}$ spacing. A magnetic anisotropy is induced by applying a magnetic field of 2 kOe during deposition. Each GMI sensor represents one sensing element. Sensing 


\section{WILEY-VCH}

elements are also connected to form arrays, which can provide information about the distribution, direction or stability of the applied force.

The nanocomposite has a maximum of $14 \%$ NWs/PDMS volume ratio, which is chosen to provide the biasing field for the GMI sensor while not adversely affecting the polymerization of the PDMS or the elasticity of the cilia. Nanocomposite cilia with diameters from $20 \mu \mathrm{m}$ to $200 \mu \mathrm{m}$ are fabricated using a master mold technique. Cilia with diameters greater than $100 \mu \mathrm{m}$ are fabricated using a poly(methyl methcrylate) (PMMA) master mold into which an array of holes with the required diameter is patterned with a $\mathrm{CO}_{2}$ laser cutter. Smaller cilia diameters are fabricated using a silicon mold prepared by conventional lithography process followed by deep reactive ion etching to form the micro holes. After casting the nanocomposite onto the surface of the GMI sensor the mold is mounted on top and the nanocomposite is cured at $90{ }^{\circ} \mathrm{C}$ for one hour with a magnetic field of $1.2 \mathrm{kOe}$ applied along the length of the cilia for NW alignment. The fabrication process is illustrated in Figure 1(b). A scanning electron microscopy image of iron NWs is shown in Figure 1(c). Transmission electron microscopy imaging is conducted to study the alignment and distribution of NWs in cilia's cross section (Figure 1(d)). The Young's modulus of the nanocomposite cilia is obtained experimentally and found to be 0.76 $\mathrm{MPa}$. The permanent magnetic properties of the nanocomposite are studied by obtaining the magnetization loops along the parallel and the perpendicular direction of $1 \mathrm{~mm}$ long and $200 \mu \mathrm{m}$ in diameter cilia using a vibrating sample magnetometer. As shown in Figure 2(a), the cilia have a magnetic anisotropy with a remanence to saturation magnetization of $93 \%$ along the vertical direction and $37 \%$ along the horizontal direction with a coercivity of $1.8 \mathrm{kOe}$.

In order to characterize the GMI sensor, a magnetic field is applied with a Helmholtz coil, while the impedance is obtained from the complex reflection coefficient measured with a network analyzer (Agilent E5061B) at a frequency of $500 \mathrm{MHz}$. A highly sensitive region is observed between 2.5 Oe to 8 Oe with an impedance peak obtained at a field of 9 Oe corresponding to 


\section{WILEY-VCH}

the anisotropy field of the $\mathrm{Ni}_{80} \mathrm{Fe}_{20}$ thin films (Figure 2(b)). The stray field of the nanocomposite cilia provides a bias field of about 3.2 Oe at which the GMI sensor has a sensitivity of $1.2 \Omega /$ Oe. The performance of the tactile sensor is investigated using different cilia geometries on $2 \times 2$ $\mathrm{mm}^{2}$ magnetic sensors to obtain results in different pressure regimes with tailored values of sensitivity, resolution, and operating range. Figure 3(a) shows a tactile sensor with $1 \mathrm{~mm}$ long and $200 \mu \mathrm{m}$ in diameter cilia arrays with 9 and 24 cilia. The sensor is characterized by applying vertical forces using a computer controlled movable plate and collecting the impedance response with a network analyzer similar to the GMI sensor characterization setup (Figure 3(b)). As shown in Figure 3(c), the sensor with 9 cilia can detect vertical forces up to $200 \mathrm{mN}$ (50 $\mathrm{kPa})$ with a high resolution of $0.9 \mathrm{mN}(0.23 \mathrm{kPa})$ and a sensitivity of $15 \mathrm{~m} \Omega / \mathrm{mN}(60 \mathrm{~m} \Omega / \mathrm{kPa})$. Thereby, the sensor operates at an extremely low power consumption of $80 \mathrm{nW}$. The maximum impedance change of the sensor is about $0.8 \Omega$ when fully deflecting the cilia. The 24 cilia arrangement tactile sensor shows a response in a larger range up to $680 \mathrm{mN}(170 \mathrm{kPa})$ with a resolution of $3.5 \mathrm{mN}(0.88 \mathrm{kPa})$ and a sensitivity of $4 \mathrm{~m} \Omega / \mathrm{mN}(16 \mathrm{~m} \Omega / \mathrm{kPa})$. A higher saturation impedance change of $0.86 \Omega$ is observed due to the presence of a larger volume of magnetic NWs affecting the GMI sensor.

The capability of the sensor to detect the texture of objects is verified by moving a PMMA object that contains $200 \mu \mathrm{m}$ deep grooves with widths of $2 \mathrm{~mm}$ and $4 \mathrm{~mm}$ horizontally across the cilia sensors with a speed of $0.4 \mathrm{~mm} / \mathrm{s}$. The sensor is operated at $500 \mathrm{MHz}$ and the signal is measured using a real time spectrum analyzer (Tek RSA 6100A). As shown in Figure 3(d), the sensor signal closely follows the pattern of the textured object with $255 \mu \mathrm{V}$ change of the voltage amplitude between the high and low features of the moving object.

To achieve the human skin's capability to "feel" irritants (such as the effects of a fly landing), ${ }^{[15]}$ sensors for a low pressure regime are required. To this end, an array of 2500 smaller cilia each with $10 \mu \mathrm{m}$ in diameter and $50 \mu \mathrm{m}$ in length is fabricated and realized on the GMI sensor. The sensor is tested by applying vertical forces up to $3.5 \mathrm{mN}(0.85 \mathrm{kPa})$ (Figure 3(e)). A maximum 


\section{WILEY-VCH}

impedance change of $0.4 \Omega$ is observed with a sensitivity of $214 \mathrm{~m} \Omega / \mathrm{mN}(856 \mathrm{~m} \Omega / \mathrm{kPa})$ and an ultra-high resolution of $0.065 \mathrm{mN}(16 \mathrm{~Pa})$.

The tactile nanocomposite cilia sensor concept can also be realized on flexible substrates. We used Kapton for this purpose and fabricated the $4 \mathrm{~mm}^{2}$ meander shape GMI sensor and 24 nanocomposite cilia with $1 \mathrm{~mm}$ in length and $200 \mu \mathrm{m}$ in diameter on top of it as shown in Figure 3(f). GMI sensors maintain their high sensitivity when fabricated on Kapton and only show a shift of the peak value related to a change of the anisotropy field. ${ }^{[34]}$ A real time measurement similar to the textured object detection experiment is conducted using a flexible sensor placed directly above the artery of the wrist. With the cilia in contact with the skin, mechanical plethysmography is realized, enabling the detection of the heart rate. ${ }^{[35]}$ As shown in Figure $3(\mathrm{~g})$, a heart rate of 85 beats $\mathrm{min}^{-1}$ is found from the signal peaks of about $100 \mathrm{uV}$ caused by the blood pressure waves.

The versatility of our sensor concept enables operation not only in air but also in liquids without modifications. This is demonstrated in Figure 4(a), which shows the response of a tactile sensor with 24 pillars tested in air and water.

A distinct feature of the cilia tactile sensor is its ability to measure shear forces as arising from fluid flow. To this end, the sensors have been implemented in a microfluidic channel using 500 $\mu \mathrm{m}$ long cilia with different aspect ratios. ${ }^{[33]}$ In case of water flow, sensors with $100 \mu \mathrm{m}$ diameter cilia operate up to $7.8 \mathrm{~mm} / \mathrm{s}(0-15 \mathrm{kPa})$ with a maximum sensitivity and resolution of $0.9 \Omega /(\mathrm{mm} / \mathrm{s})$ and $15 \mu \mathrm{m} / \mathrm{s}$, respectively. Increasing the diameter of the cilia to $250 \mu \mathrm{m}$ increases the operating range to $10 \mathrm{~mm} / \mathrm{s}(0-20 \mathrm{kPa})$ with a maximum sensitivity and resolution of 0.17 $\Omega /(\mathrm{mm} / \mathrm{s})$ and $79 \mu \mathrm{m} / \mathrm{s}$, respectively (Figure $4(\mathrm{~b})$ ). It is worth noting that the sensor operates at extremely low power consumption of about $80 \mathrm{nW}$.

In summary, novel permanent magnetic and highly elastic nanocomposite cilia structures have been realized on top of magnetic GMI sensors to enable biomimetic tactile sensing. The permanent magnetic properties of the nanocomposite are achieved by using Fe NWs, which 


\section{WILEY-VCH}

generate a stray field that is detected by the GMI sensor and which is a function of the cilia's deflection. This property of the NWs is crucial because it avoids the need for an external magnetic field source for both magnetizing the nanocomposite and biasing the GMI sensor. GMI sensors are used because of their high sensitivity and simple fabrication, and it is worth to note that they could be implemented as wireless devices which could be utilized to realize passive and remote sensors. ${ }^{[31]}$ Different cilia configurations were designed, confirming the possibility to easily control the sensors' performance with the cilia's arrangements or dimensions. The magnetic nanocomposite cilia sensor concept works on both rigid as well as flexible substrates and the sensors can be used in air or water without any modifications. A specific feature of the cilia tactile sensor is its ability to measure flow, which is unique in the realm of tactile sensors.

The microfabrication process and magnetic operation principle enable a high degree of integration, which together with the extremely low power consumption make the artificial cilia sensor an attractive solution for many applications.

\section{Experimental}

NWs Fabrication: Fe NWs are fabricated by the electrodeposition in self-ordered anodic alumina (AAO) membranes that are synthesized by a two-step anodization process using oxalic acid. Prior to anodization, high-purity (99.999\%) aluminum disks are cleaned in acetone path using ultrasound and then an electro-polishing step is followed using a mixture of perchloric acid and ethanol $(\mathrm{HClO} 4: \mathrm{C} 2 \mathrm{H} 5 \mathrm{OH}=1: 4$ for 2 minutes. The first anodization is performed using oxalic acid solution as electrolyte at $2{ }^{\circ} \mathrm{C}$ temperature and an anodization voltage of $40 \mathrm{~V}$ for 24 hours. Following the first anodization, the sample is immersed in chrome solution at $40{ }^{\circ} \mathrm{C}$ temperature for 12 hours to dissolve the Alumina membrane. The second anodization is performed for 10 hours to form a $20 \mu \mathrm{m}$ thick Alumina membrane with highly ordered pores. Underneath the nanopores, an alumina thin layer present so a voltage reduction step is 


\section{WILEY-VCH}

conducted using the same conditions of the anodization process to form dendrites that allow interfacing the nanopores with Aluminum to provide electrical connection for the electrodeposition process. Polycrystalline Fe NWs are then electrodeposited from an aqueous Watts bath of $45 \mathrm{~g} \mathrm{~L}^{-1} \mathrm{FeSO}_{4}, 30 \mathrm{~g} \mathrm{~L}^{-1}$ Boric acid $\left(\mathrm{H}_{3} \mathrm{BO}_{3}\right)$ and $1 \mathrm{~g} \mathrm{~L}^{-1}$ Ascorbic acid $\left(\mathrm{C}_{6} \mathrm{H}_{8} \mathrm{O}_{6}\right)$. Pulsed electrodeposition is carried out at room temperature resulting in $6 \mu \mathrm{m}$ long and $35 \mathrm{~nm}$ in diameter NWs. The NWs is then released from the membrane by etching Alumina using chrome solution for 24 hours at $40{ }^{\circ} \mathrm{C}$ with constant agitation. The NWs are then washed and kept in Ethanol.

Nanocomposite Cilia Fabrication: The nanocomposite is prepared by mixing iron NWs dispersed in Sodium dodecyl sulfate (SDS) surfactant in PDMS (Sylgard 184 Silicone Elastomer, Dow Corning Corporation) that is used as the polymeric matrix.

Nanocomposite cilia are fabricated using a master mold technique. The long cilia (e.g. $1 \mathrm{~mm}$ ) were fabricated using a PMMA master mold with the thickness corresponding to the cilia length into which an array of holes with $200 \mu \mathrm{m}$ in diameter is patterned with a $\mathrm{CO}_{2}$ laser cutter (Universal PLS6.75). Cilia with smaller diameters are fabricated using a silicon mold prepared by a photolithography process followed by deep reactive ion etching to form the microholes. After spinning and curing a $2 \mu \mathrm{m}$ thin PDMS layer on the GMI sensor to provide electrical insolation and increase the adhesion of the cilia to the substrate, the nanocomposite is casted onto the surface of the GMI sensor and the master mold is mounted on top with aligning the holes along the GMI sensor using a microscope. This structure is then placed in a desiccator for 1 hour to remove trapped air bubbles and assist in filling the pores. During that time a homogeneous magnetic field is applied to define the anisotropy by aligning the NWs in the desired direction. Next, the nanocomposite is cured at $90{ }^{\circ} \mathrm{C}$ for one hour while keeping the external magnetic field, forming the cilia with aligned NWs on top of the GMI sensor. After releasing the cured cilia, they are fully magnetized by applying a magnetic field of $10 \mathrm{kOe}$. 


\section{WILEY-VCH}

GMI Sensor Fabrication: The GMI sensor is designed as a meander shape confined within a 4 $\mathrm{mm}^{2}$ sensing area, and has a width of $200 \mu \mathrm{m}$ with spacing of $200 \mu \mathrm{m}$. The GMI sensor is composed of $200 \mathrm{~nm}$ thick $\mathrm{Cu}$ layer sandwiched by two $100 \mathrm{~nm}$ thick $\mathrm{Ni}_{80} \mathrm{Fe}_{20}$ layers. The sensor is patterned onto a rigid $\mathrm{Si}$ substrate with $500 \mathrm{~nm} \mathrm{SiO}_{2}$ or on a flexible kapton substrate using standard lithography and the materials are deposited by e-beam evaporation with a constant magnetic field of 100 Oe applied in the transverse direction to induce a uniaxial magnetic anisotropy, creating a magnetically sensitive axis in the longitudinal direction, and then followed by a lift-off process.

\section{Acknowledgements}

Research reported in this publication was supported by the King Abdullah University of Science and Technology (KAUST).

Received: ((will be filled in by the editorial staff))

Revised: ((will be filled in by the editorial staff)) Published online: ((will be filled in by the editorial staff))

[1] Y. Park, B. Chen, R. J. Wood, IEEE Sens. J. 2012, 12, 2711.

[2] T. Someya, T. Sekitani, S. Iba, Y. Kato, H. Kawaguchi, T. Sakurai, Proc. Natl. Acad. Sci. USA 2004, 101, 9966.

[3] K. Takei, T. Takahashi, J. C. Ho, H. Ko, A. G. Gillies, P. W. Leu, R. S. Fearing, A. Javey, Nature Mater. 2010, 9, 821.

[4] S. C. B. Mannsfeld, B. C. -K. Tee, R. M. Stoltenberg, C. V. H.-H. Chen, S. Barman, B. V. O. Muir, A. N. Sokolov, C. Reese, Z. Bao, Nature Mater. 2010, 9, 859. 


\section{WILEY-VCH}

[5] G. Schwartz, B. C. -K. Tee, J. Mei, A. L. Appleton, D. H. Kim, H. Wang, Z. Bao, Nature Comm. 2013, 4, 1859.

[6] W.-H. Yeo, Y.-S. Kim, J. Lee, A. Ameen, L. Shi, M. Li, S. Wang, R. Ma, S. H. Jin, Z. Kang, Y. Huang, J. A. Rogers, Adv. Mater. 2013, 25, 2773.

[7] D. Kim, N. Lu, R. Ghaffari, Y. Kim, S. P. Lee, L. Xu, J. Wu, R.-H. Kim, J. Song, Z. Liu, J. Viventi, B. de Graff, B. Elolampi , M. Mansour, M. J. Slepian, S. Hwang, J. D. Moss, S.-M. Won, Y. Huang, B. Litt, J. A. Rogers, Nature Mater. 2011, 10, 316.

[8] M. Ying, A. P. Bonifas, N. Lu, Y. Su, R. Li, H. Cheng, A. Ameen, Y. Huang and J. A Rogers, Nanotech. 2012, 23, 344004.

[9] J. Kim, M. Lee, H. J. Shim, R. Ghaffari, H. R. Cho, D. Son, Y. H. Jung, M. Soh, C. Choi, S. Jung, K. Chu, D. Jeon, S. Lee, J. H. Kim, S. H. Choi, T. Hyeon, D. Kim, Nature Comm. 2014, 5, 5747.

[10] T. Takahashi, K. Takei, A. G. Gillies, R. Fearing, A. Javey, Nano Lett. 2011, 11, 5408.

[11] T. Someya, Y. Kato, T. Sekitani, S. Iba, Y. Noguchi, Y. Murase, H. Kawaguchi, T. Sakurai, Proc. Natl. Acad. Sci. USA 2005, $102,12321$.

[12] S. Gong, W. Schwalb, Y. Wang, Y. Chen, Y. Tang, J. Si, B. Shirinzadeh, W. Cheng, Nature Comm. 2014, 5, pp. 1.

[13] B. Zhu, Z. Niu, H. Wang, W. R. Leow, H. Wang, Y. Li, L. Zheng, J. Wei, F. Huo, and X. Chen, Small 2014, 10, 3625.

[14] O. Yilmazoglu, A. Popp, D. Pavlidis, J. Schneider, D. Garth, F. Schüttler G.

Battenberg, Nanotech. 2012, 23, 085501.

[15] S. Mannsfeld, B. Tee, R. Stoltenberg, C. Chen, S. Barman, B. Muir, A. Sokolov, C. Reese Z. Bao, Nature Mater. 2010, 9, 859.

[16] C. Pang, J. H. Koo, A. Nguyen, J. M. Caves, M. Kim, A. Chortos, K. Kim, P. J. Wang, J. B. Tok, Z. Bao, Adv. Mater. 2015, 27, 634. 


\section{WILEY-VCH}

[17] C. Pang, G.-Y. Lee, T.-i. Kim, S. M. Kim, H. N. Kim, S.-H. Ahn, K.-Y. Suh, Nat. Mater. 2012, 11,795 .

[18] L. Viry, A. Levi , M. Totaro, A. Mondini, V. Mattoli, B. Mazzolai, and L. Beccai, Adv. Mater. 2014, 26, 2659.

[19] S. Yun, S. Park, B. Park, Y. Kim, S. K. Park, S. Nam, K. Kyung, Adv. Mater. 2014, 26,4474 .

[20] C. Yeom, K. Chen, D. Kiriya, Z. Yu, G. Cho, A. Javey, Adv. Mater. 2015, 27, 1561.

[21] C. Choong, M. Shim, B. Lee, S. Jeon, D. Ko, T. Kang, J. Bae, S. H. Lee, K. Byun, J. Im, Y. J. Jeong, C. E. Park, J. Park, U. Chung, Adv. Mater. 2014, 26, 3451.

[22] N. T. Tien, S. Jeon, D. Kim, T. Q. Trung, M. Jang, B. Hwang, K. Byun, J. Bae, E. Lee, J. B. Tok, Z. Bao, N. Lee, J. Park, Adv. Mater. 2014, 26, 796.

[23] D. Son, J. Lee, S. Qiao, R. Ghaffari, J. Kim, J. E. Lee, C. Song, S. J. Kim, D. J. Lee, S. W. Jun, S. Yang, M. Park, J. Shin, K. Do, M. Lee, K. Kang, C. S. Hwang, N. Lu, T. Hyeon, D.-H. Kim, Nature Nanotechnol. 2014, 9, 397.

[24] S. Peleshanko, M. D. Julian, M. Ornatska, M. McConney, M. LeMieux, N. Chen, C. Tucker, Y. Yang, C. Liu, J. A. C. Humphrey, V. V. Tsukruk, Adv. Mater. 2007, 19, 2903.

[25] M. Burrows, G. Sutton, Science 2013, 341, 1254.

[26] J. Casas, T. Steinmann, G. Krijnen, J. R. Soc. Interface 2010, 7, 1487.

[27] M. M. Lagarde, M. Drexl, V. A. Lukashkina, A. N. Lukashkin, I. J. Russell, Nat. Neurosci. 2008, 7, 746.

[28] L. Panina, K. Mohri, Sens. Actuat. A 2000, 81, 71.

[29] B. Li, N. P. Salem, I. Giouroudi, J. Kosel, J. Appl. Phys. 2012, 111, $07 \mathrm{E} 514$.

[30] B. Li, J. Kosel, J. Appl. Phys. 2011, 109, 07E519.

[31] B. Li, A. Morsy, J. Kosel, IEEE Trans. Mag. 2012, 11, 4324.

[32] M. Song, W. Song, H. Bi, J. Wang, W. Wu, J. Sun, M. Yu, Biomat. 2010, 31, 1509.

[33] A. Alfadhel, B. Li, A. Zaher, O. Yassine, J. Kosel, Lab Chip 2014, 14, 4362. 
[34] B. Li, M. N. Kavaldzhiev, J. Kosel, J. of Mag. and Mag. Mater. 2015, 378, 499.

[35] E. Kaniusas, H. Pfützner, L. Mehnen, J. Kosel, J.C. Téllez-Blanco, G. Varoneckas, A.

Alonderis, T. Meydan, M. Vázquez, M. Rohn, A.M. Merlo, B. Marquard, IEEE Sensors

Journal. 2006, 6, 3, 819.
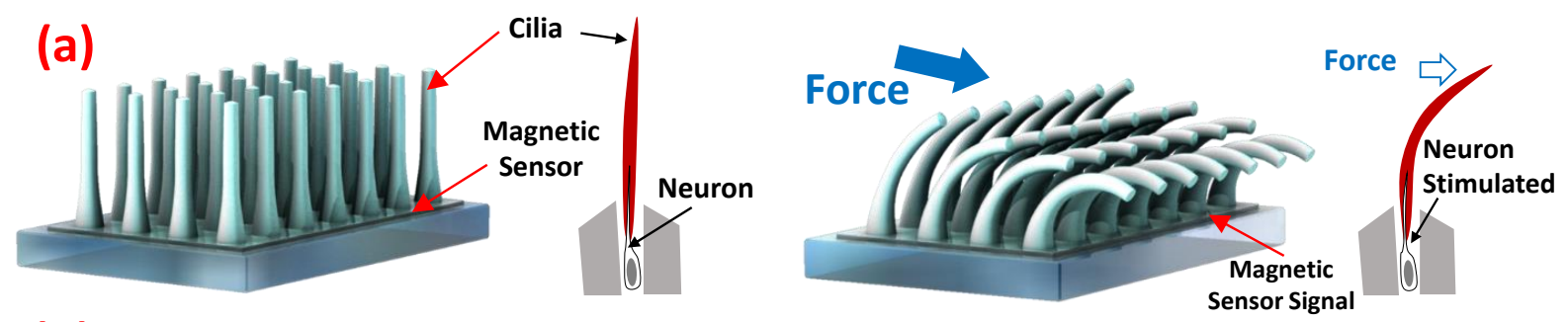

(b)
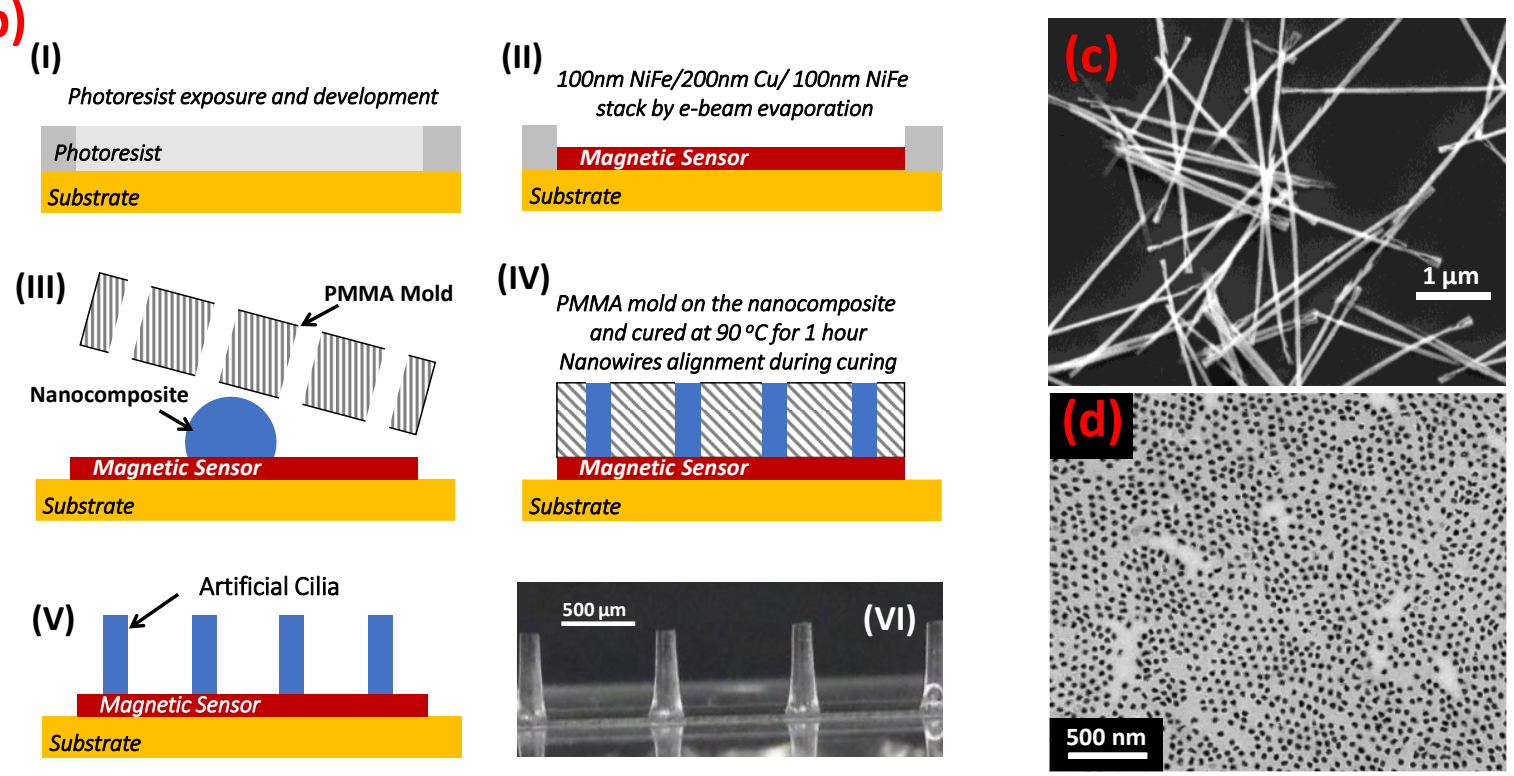

Figure 1. (a) Illustration of the nanocomposite cilia tactile sensor. The sensor is made of permanent magnetic nanocomposite cilia integrated on a magnetic sensor that mimics the neuron in natural cilia. When the cilia get deflected by external forces such as fluid flow or hand touch, the stray field at the magnetic sensor changes, which also changes its impedance.

(b) Fabrication process of the tactile sensor. (I,II) A GMI sensor is fabricated by depositing $100 \mathrm{~nm}$ permalloy/ $200 \mathrm{~nm} \mathrm{Cu} / 100 \mathrm{~nm}$ permalloy by e-beam evaporation and it is patterned using a standard lithography process. (II-V) Cilia are fabricated using a PMMA mold. (VI) Cilia with $500 \mu \mathrm{m}$ in length and $100 \mu \mathrm{m}$ in diameter. (c) Scanning electron microscopy image of dispersed NWs prior to the nanocomposite fabrication. (d) Transmission electron microscopy image of the cilia's cross section showing the distribution of the NWs in the nanocomposite. 
WILEY-VCH
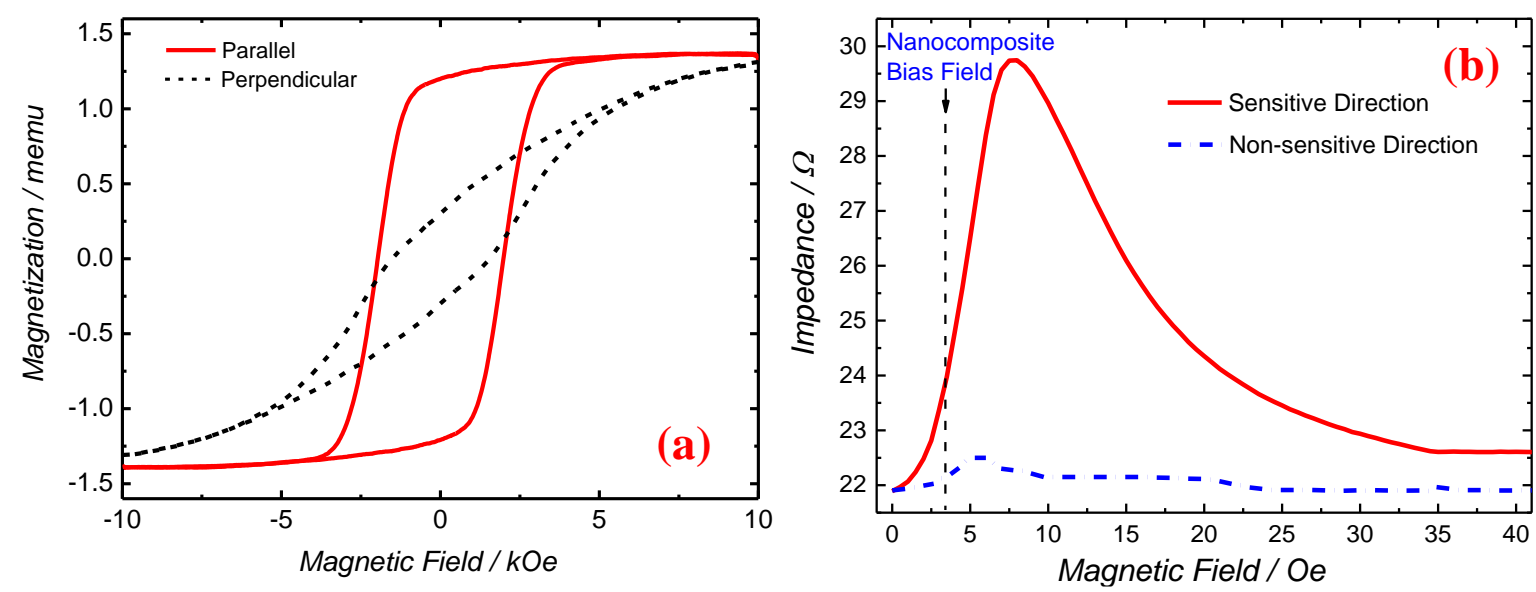

Figure 2. (a) Magnetization loops of magnetic cilia obtained with a vibrating sample magnetometer in the parallel and perpendicular direction of the cilia with vertically aligned NWs. (b) Impedance response of the GMI sensor to an external magnetic field applied at 500 $\mathrm{MHz}$ operating frequency. 

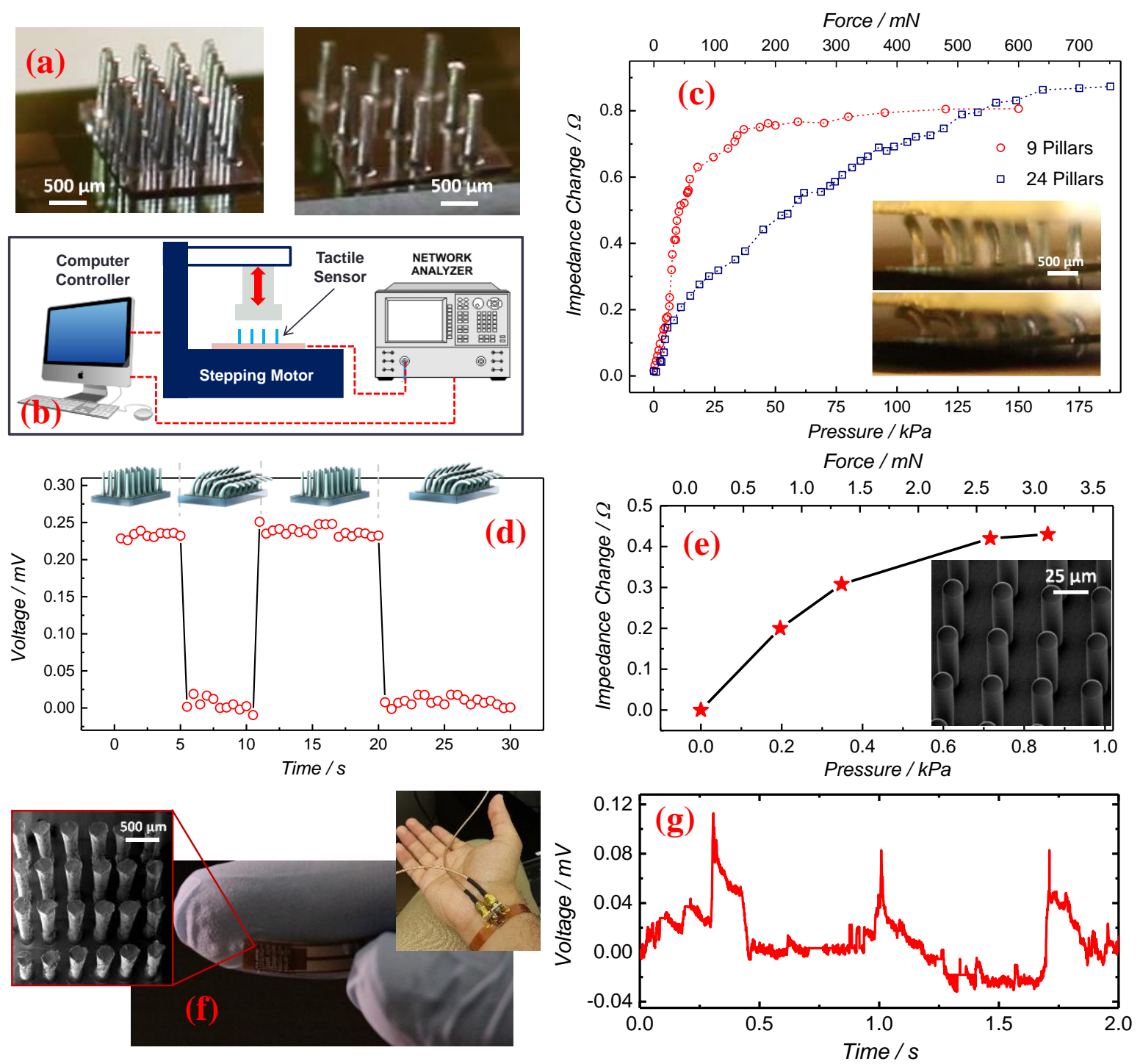

Figure 3. (a) Arrays of 9 and 24 cilia. (b) Tactile sensor test setup. (c) Tactile sensor impedance response for two cilia configurations: 9 and 24 cilia array. Inset: Deflection mechanism of cilia sensor with a vertical force. (d) Dynamic response for texture measurement. (e) Force response of a sensor with 2500 cilia with $10 \mu \mathrm{m}$ in diameter, $50 \mu \mathrm{m}$ long, and $25 \mu \mathrm{m}$ separation. Inset: SEM image of the fabricated cilia array. (f) A photograph of a flexible tactile sensor with an inset showing an SEM image of the cilia, and a photograph

showing the flexible sensor attached to the skin above the artery of the wrist. $(\mathrm{g})$

Measurement of the heart rate $\left(85\right.$ beats $\left.\mathrm{min}^{-1}\right)$ through the signal caused by the blood pressure waves, which cause bending of the cilia. 


\section{WILEY-VCH}
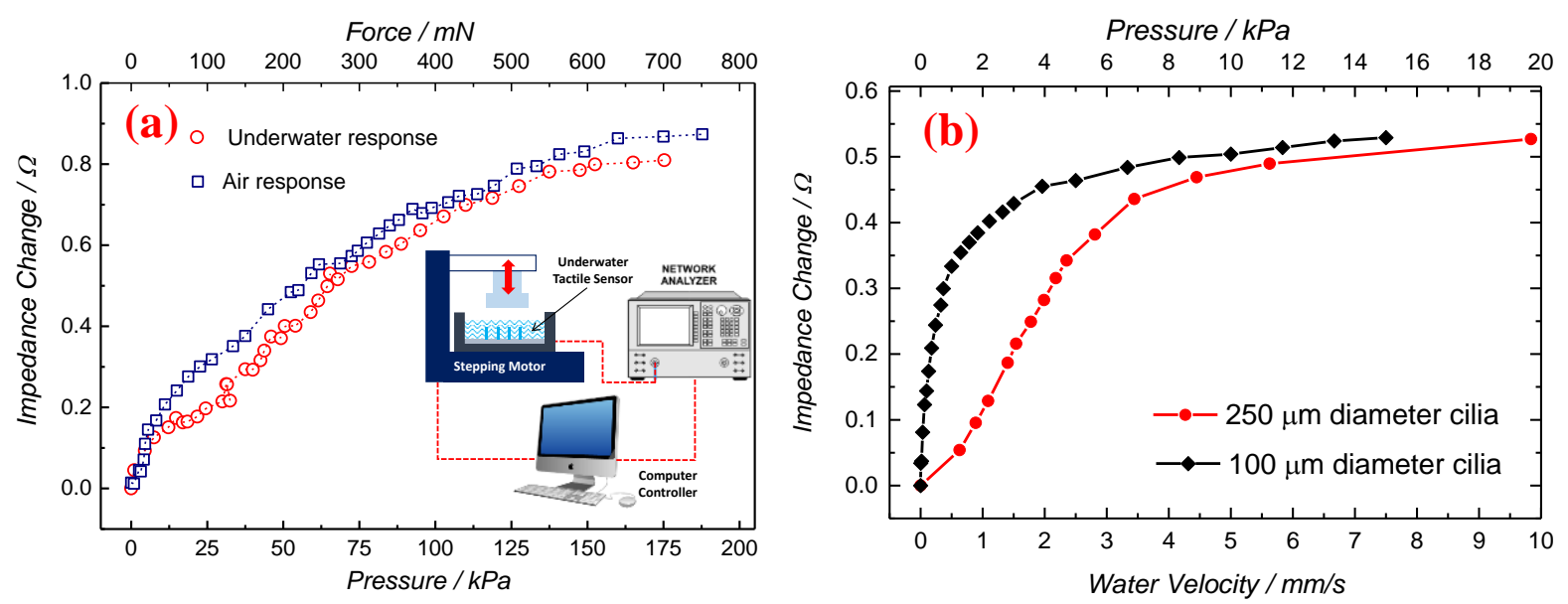

Figure 4. (a) Underwater performance of a tactile sensor with $1 \mathrm{~mm}$ long and $200 \mu \mathrm{m}$ in diameter cilia. Inset: Test setup for underwater experiment. (b) Water flow sensor response of sensors with cilia that are $500 \mu \mathrm{m}$ long and have a diameter of $100 \mu \mathrm{m}$ and $250 \mu \mathrm{m}$. 


\section{WILEY-VCH}

\section{The table of contents entry}

A multifunctional biomimetic nanocomposite tactile sensor is developed that can detect shear and vertical forces, feel texture, and measure flow with extremely low power consumption. The sensor's high performance is maintained within a wide operating range that can be easily adjusted. The concept works on rigid and flexible substrates and the sensors can be used in air or water without any modifications.

\section{Keyword}

tactile sensors, nanocomposites, nanowires, magnetic sensors, cilia
A. Alfadhel, Prof. J. Kosel*

\section{Nanocomposite Cilia Tactile Sensor}

ToC figure

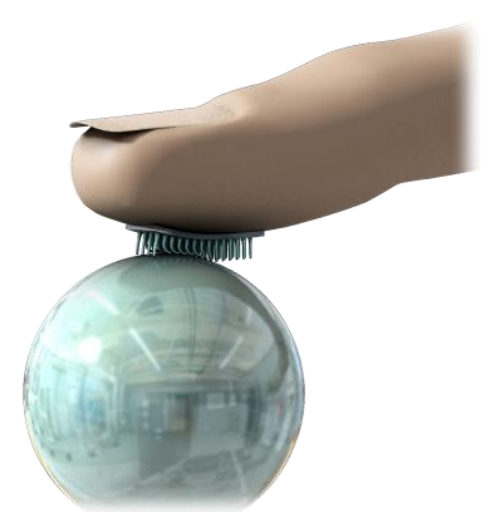




\section{WILEY-VCH}

((Supporting Information can be included here using this template))

Copyright WILEY-VCH Verlag GmbH \& Co. KGaA, 69469 Weinheim, Germany, 2013.

Supporting Information

Nanocomposite Cilia Tactile Sensor

Ahmed Alfadhel, and Jürgen Kosel*

((Please insert your Supporting Information text/figures here. Please note: Supporting Display items, should be referred to as Figure S1, Equation S2, etc., in the main text...) 